\title{
Transatlantica
}

Revue d'études américaines. American Studies Journal

«Spirit of the dead, rise up! [...] and claim your story ». Représentation de l'esclavage et esthétique de la résistance dans Sankofa (Haile Gerima, 1993)

\section{Claire Dutriaux}

\section{(2) OpenEdition}

\section{Journals}

Édition électronique

URL : https://journals.openedition.org/transatlantica/12638

DOI : 10.4000/transatlantica. 12638

ISSN : 1765-2766

Éditeur

Association française d'Etudes Américaines (AFEA)

Référence électronique

Claire Dutriaux, « « Spirit of the dead, rise up! [...] and claim your story ». Représentation de l'esclavage et esthétique de la résistance dans Sankofa (Haile Gerima, 1993) », Transatlantica [En ligne], 1| 2018, mis en ligne le 18 septembre 2019, consulté le 01 février 2023. URL : http://journals.openedition.org/ transatlantica/12638; DOI : https://doi.org/10.4000/transatlantica.12638

Ce document a été généré automatiquement le 1 février 2023

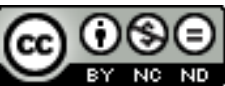

Creative Commons - Attribution - Pas d'Utilisation Commerciale - Pas de Modification 4.0 International - CC BY-NC-ND 4.0

https://creativecommons.org/licenses/by-nc-nd/4.0/ 


\title{
«Spirit of the dead, rise up! [...] and claim your story ». Représentation de l'esclavage et esthétique de la résistance dans Sankofa (Haile Gerima, 1993)
}

\author{
Claire Dutriaux
}

Haile Gerima, réalisateur africain-américain né en 1946 en Éthiopie dans une famille chrétienne, partit étudier aux États-Unis à l'âge de vingt et un ans (Ukadike, 2002 253). Il s'installa tout d'abord à Chicago, où il suivit les cours de théâtre de la Goodman School of Drama, puis en 1969 s'inscrivit dans le Master of Fine Arts Film Program de l'université de Californie à Los Angeles (UCLA), ouvert à tout étudiant issu de minorités ("Story of LA Rebellion»). Il y rencontra d'autres réalisateurs noirs américains indépendants tels Julie Dash, Larry Clark et Charles Burnett, soit le groupe de réalisateurs africains et africains-américains que l'historien du film Clyde Taylor surnomma par la suite la LA Rebellion (Taylor; voir également Field, Horak et Stewart). Ces réalisateurs et réalisatrices, de la même génération, cherchèrent à créer un cinéma noir américain indépendant, dont les codes et les conventions n'étaient pas ceux traditionnellement utilisés dans le cinéma hollywoodien, afin de rendre compte de l'expérience unique des communautés noires dans les Amériques, en Europe et en Afrique. En parallèle, les études de Gerima à UCLA lui permirent de se familiariser avec l'œuvre de Frantz Fanon et de W.E.B. Du Bois, ainsi qu'avec le "troisième cinéma " d'Afrique et d'Amérique latine (Ukadike, 2002 254). Haile Gerima a eu, en même temps que sa carrière de réalisateur et de producteur, une carrière d'enseignant à l'université, qui lui a permis de rester indépendant et de financer en partie ses films; il est toujours professeur de cinéma à Howard University, à Washington, DC. Dans la plupart des entretiens qu'il a accordés, il s'est identifié à la fois comme Africain et comme Noir américain : "Even if I were to return to Africa, I would always protect my direct links with black America. It has given me the courage to discover myself. [...] At first, I 
considered myself Ethiopian rather than connected to black America. Black America helped to humanize me " (cité par Reid 108). En tant que membre à part entière du mouvement de la LA Rebellion, Haile Gerima a souhaité réinventer dans Sankofa (1993) le discours hollywoodien dominant sur l'esclavage, qui exposait la thématique en termes binaires. Avant Sankofa, les films hollywoodiens traitant de l'esclavage présentaient les Noirs américains soit comme des victimes ayant besoin d'être secourues par des personnages blancs empathiques - par exemple dans les adaptations de La Case de l'oncle Tom (Uncle Tom's Cabin), notamment celle de Harry Pollard en 1927 -, soit comme des êtres détestables et mauvais qu'il convenait de faire disparaître de l'écran pour restaurer l'unité de la nation américaine blanche, tels Gus (Walter Long) et Silas Lynch (George Siegmann) dans Naissance d'une nation (The Birth of a Nation, D.W. Griffith, 1915). L'expérience de l'esclavage, du point de vue de personnages africains-américains, a été représentée dans une moindre mesure dans le cinéma d'exploitation, comme dans Mandingo (Richard Fleischer, 1975), mais elle a surtout été rendue possible à la télévision dans Racines (Roots, 1977), mini-série au succès planétaire.

2 À l'instar d'un Oscar Micheaux, qui dès les années 1910 a cherché à produire d'autres images de la population noire américaine que celles du film de D.W. Griffith, notamment dans Within Our Gates (1920) et Body and Soul (1925), sans toutefois réussir toujours à se départir du discours filmique dominant, Haile Gerima et les réalisateurs de la LA Rebellion ont souhaité produire d'autres discours, qui puissent rendre compte de la véritable expérience de la diaspora africaine, dans les centres urbains en particulier. Ed Guerrero écrit ainsi :

the "black independent cinema movement" inspired by the films of universitytrained black filmmakers of the 1970s laid a clear political, philosophical, and aesthetic foundation for an ongoing cinematic practice that challenges Hollywood's hegemony over the black image. The films of Charles Burnett [...], Julie Dash [...], Bill Woodberry [...], and Haile Gerima [...] have all contributed to creating an emergent, decolonizing, antiracist cinema that in its images, sounds, aesthetics, and modes of production has attempted to reconstruct the world on the screen from black points of view cast in liberating images and new paradigms. (Guerrero 137)

Fruit d'une coproduction entre Mypheduh Films, la compagnie de production de Gerima, et le Ghana et le Burkina Faso, Sankofa se distingue du cinéma hollywoodien non seulement en raison de ses multiples connexions entre les Amériques et l'Afrique et de son contenu singulier, mais également parce que Gerima s'est volontairement inspiré des stratégies des cinéastes noirs américains des années 1920, notamment Oscar Micheaux $^{1}$, mais aussi de Spike Lee avec Malcolm X (1992), pour financer et distribuer son film lorsqu'il n'essuya que des refus des distributeurs américains. Gerima identifiait ces refus comme de la censure institutionnelle, qu'il convenait de contourner en employant ses propres méthodes de financement et de distribution: "In America, slavery is a very sensitive topic. The moment I wanted to make Sankofa (1993), my credentials in the USA vanished because I was venturing into forbidden territory. The resource centers were closed to me. I couldn't get funding " (cité par Reid 115). Gerima s'adressa alors à des investisseurs privés et à des associations sur d'autres continents. Quand vint le moment de distribuer le film, il parcourut les États-Unis afin de le montrer dans des églises, des centres communautaires, des festivals et des cinémas loués pour l'occasion, tout en l'accompagnant de conférences. Gerima sut s'entourer de personnalités africaines-américaines influentes, tel le joueur de basketball Magic Johnson, propriétaire de multiplexes, pour distribuer son œuvre (Crémieux, 2009). Le bouche à oreille permit au film de connaître le succès : il généra plus de 2,5 millions de 
dollars de recettes, soit deux fois et demie le montant initialement investi pour le produire, ce qui permit à Gerima d'installer sa propre maison de production (Mypheduh Films) ainsi que son magasin de vidéo (Sankofa) à Washington, DC. Variety, revue de l'industrie hollywoodienne par excellence, consentit même à écrire quelques lignes sur le phénomène de l'autodistribution (Weiner). Gerima s'affranchit néanmoins de l'héritage d'Oscar Micheaux, dont il étudia les films à UCLA, en se concentrant sur un passé esclavagiste que Micheaux ne montrait pas dans ses films, centrés sur l'expérience urbaine et contemporaine des Noirs américains, et en mettant en lumière le concept de diaspora noire.

3 Sankofa raconte l'histoire de Mona (Oyafunmike Ogunlano), jeune mannequin américaine insouciante venue travailler avec son photographe blanc au Ghana. Lors d'une séance photo dans le fort d'Elmina, qui servait de prison d'esclaves au temps du commerce triangulaire, Mona croise Sankofa (Kofi Ghanaba), le gardien spirituel du fort, qui l'exhorte à se tourner vers son passé et la menace : «sankofa » est le nom d'un oiseau de la mythologie akan dont la tête est tournée vers l'arrière pour signifier que "pour avancer, il faut revenir en arrière ». Alors que Mona explore le fort, elle se trouve propulsée en un temps et un lieu différents. Dans le sous-sol du fort, elle voit la traite négrière avant d'être violemment dénudée et marquée au fer rouge : elle devient, ou redevient, Shola, une esclave domestique envoyée sur la plantation Lafayette où est cultivée la canne à sucre. Le film narre alors la vie sur la plantation, rythmée par la dureté du travail de coupe, les coups de fouet, la violence physique et psychologique exercée par les maitres et le prêtre blancs, les discordes entre esclaves domestiques et esclaves des champs, les viols des esclaves noires commis par les Blancs, mais aussi la construction d'une communauté solidaire avec son propre folklore et la naissance de la rébellion et de la résistance qui permettent aux Noirs d'exister dans un monde qui veut nier leur humanité. Les moments fondateurs de cette résistance sont liés à trois personnages centraux: Shango (Mutabaruka), l'amant de Shola, rebelle par essence; Nunu (Alexandra Duah), qui symbolise la Mère Afrique et le lien entre l'Afrique et l'Amérique ; et son fils Joe (Nick Medley), mulâtre aux yeux bleus né du viol de sa mère par un maitre blanc. Ces trois personnages vont conduire Shola, chacun à leur manière, à son acte final de résistance (la mise à mort du maitre blanc), acte qui lui permet d'exister et d'accomplir son voyage spirituel vers la Mère Afrique. À la fin du film, Shola s'envole dans les airs tel un oiseau sankofa et revient au fort ghanéen sous les traits de Mona, qui, désormais pleinement consciente de son passé, peut regarder vers l'avenir. Le film évoque en partie le concept de double conscience élaboré par W.E.B. Du Bois dans The Souls of Black Folk, tout en lui accordant une dimension supplémentaire puisque, dans Sankofa, la double conscience implique une connaissance du passé diasporique :

One ever feels his two-ness,-an American, a Negro; two souls, two thoughts, two unreconciled strivings; two warring ideals in one dark body, whose dogged strength alone keeps it from being torn asunder.

The history of the American Negro is the history of his strife,--this longing to attain self-conscious manhood, to merge his double self into a better and truer self. In this merging he wishes neither of the older selves to be lost. (Du Bois 3-4)

4 La résistance comme outil d'accession à la conscience, à l'existence, tel est le concept central du film, qui s'exprime tout autant dans la narration que dans la forme. Sankofa crée une esthétique de la résistance pour faire jouer le concept sur plusieurs niveaux, narratif et méta-narratif : la résistance des esclaves exprime dans le même temps une 
résistance aux tropes du cinéma hollywoodien, ce qui permet l'expression d'un cinéma noir américain avec son esthétique propre, ainsi décrite par Ed Guerrero: "the oral tradition, the complex integration of black music into both soundtrack and narrative, and the expression of a black social reality that transcends or shatters Hollywood fantasy » (Guerrero 138). Ce concept de résistance, idéologique et formelle, est exprimé dans la narration et dans la sémiotique du film, dans un mouvement qui décloisonne l'imaginaire en renversant les images, par l'usage de l'écriture symbolique, de la même manière que l'oiseau sankofa regarde en arrière pour se diriger vers l'avenir de la communauté noire américaine. Si le film a déjà fait l'objet de nombreuses études, notamment du point de vue idéologique (Letort), historique et historiographique (Kandé), et si les films des réalisateurs issus de la LA Rebellion ont été analysés à l'aune de la question raciale (Wall et Martin), la dimension esthétique et sémiotique de Sankofa a rarement été prise en compte. Or l'esthétique de la résistance que crée Haile Gerima participe pleinement au discours historique et idéologique du film.

\section{Décloisonner les esprits}

5 Dans Sankofa, Haile Gerima cherche à décloisonner un imaginaire noir américain qui a été contraint par l'esclavage. La narration du film désoriente, car elle n'est pas complètement linéaire, notamment au début et à la fin du film. Le montage est fréquemment haché par des coupes sur des gros plans d'oiseaux en vol ou sur un vautour, introduits par des fondus qui ne sont pas enchaînés. Parfois les musiques et les voix s'entremêlent, sans qu'il soit possible de les distinguer. La séquence du prologue est emblématique de ce montage dense, avec en premier lieu une focalisation de la caméra en gros plan d'abord sur un oiseau sankofa, puis sur une statue d'Africain au tambour ; un plan juxtaposant une image de soleil couchant et le fort ghanéen avec le sorcier jouant du tambour ; une succession de plans juxtaposant tour à tour les cannes à sucre et des plans de plus en plus rapprochés sur le sorcier et enfin sur le visage de Mona, ce qui crée une image triple contenant à la fois les cannes à sucre, le sorcier et Mona. Dès le prologue, Mona est tournée vers son passé d'esclave, alors que la narration du film n'a pas encore commencé : tel l'oiseau sankofa de la mythologie $\mathrm{akan}^{2}$, elle regarde vers le fort puis vers les plantations de canne à sucre où, en tant que Shola, elle sera obligée de travailler. Les séquences qui suivent le prologue rompent également avec le style d'une scène d'exposition classique (notamment dans le cinéma hollywoodien, dans lequel la cohérence de l'espace et du temps est primordiale), alternant des plans rapides avec des mouvements de caméra brutaux et des plans plus contemplatifs, entrecoupés par des plans rapprochés sur un vautour, sans qu'un lien entre ces images successives soit immédiatement perceptible. Cette accession au sens ne pourra se faire qu'au cours du film ou en regardant Sankofa une nouvelle fois, ce qui met le spectateur dans la position de l'oiseau sankofa, tout comme Mona.

Cette esthétique de la désorientation, avec des images multiples imbriquées les unes aux autres, est relayée par une narration qui, elle aussi, désoriente. Ainsi les lieux de la narration sont-ils multiples, parfois bien définis, comme le fort d'Elmina au Ghana, mais souvent imprécis, comme la plantation Lafayette sur laquelle on retrouve Shola. Le nom de la plantation, qui est aussi celui du propriétaire blanc, évoque la Louisiane, mais les champs de canne à sucre rappellent plutôt les Caraïbes, de même que la présence de montagnes et de forêts où les esclaves rebelles cherchent à se réfugier. Les 
esclaves ont de multiples origines : Nunu est identifiée comme africaine, Joe et Shola sont nés sur le territoire américain mais l'un est mulâtre et l'autre ne l'est pas, Shango est un rebelle des Caraïbes, coiffé de dreadlocks, dont le dialecte créole est systématiquement sous-titré. Il porte le nom d'une divinité vénérée dans la santería et le vaudou haïtien (Murphy 13). De même, l'époque n'est pas non plus identifiable : les séquences de la traite négrière dans le fort font penser aux XVII et XVIII siècles, mais les séquences sur la plantation suggèrent le $\mathrm{xIX}^{\mathrm{e}}$ siècle, notamment tel qu'il a été représenté dans le cinéma hollywoodien et en particulier dans Naissance d'une nation et Autant en emporte le vent (Gone with the Wind, Victor Fleming, 1939). Les lieux de tournage du film furent multiples, de la Jamaïque au Ghana en passant par la Louisiane (Kandé 92). La séquence de la transformation de Mona en Shola est un exemple parfait d'un décloisonnement des esprits qui provoque celui du spectateur: Mona semble projetée dans l'époque de la traite négrière, mais il s'agit bien de son corps qui est marqué au fer puisqu'elle crie «Wait a minute, I'm an American! ». La transformation en Shola, dans un autre temps, un autre lieu, indéfinis, est brutale et se fait sans transition.

7 Le film vise à rassembler les populations africaines et afrodescendantes, éparpillées lors de l'exil forcé de l'esclavage, en effaçant les frontières géographiques ${ }^{3}$. Le public noir, américain comme africain, doit prendre conscience, grâce aux personnages, de son histoire et de son existence en tant que diaspora. Delphine Letort remarque que la sortie du film a coïncidé avec la publication de l'ouvrage L'Atlantique noir. Modernité et double conscience (The Black Atlantic: Modernity and Double Consciousness, 1993), dans lequel l'historien Paul Gilroy présente le concept d'Atlantique noir comme une culture englobant différentes ethnies et nationalités (Letort 2). Dans son ouvrage, Gilroy emploie l'image de bateaux en mouvement entre l'Europe, l'Amérique, l'Afrique et les Caraïbes pour exprimer l'idée de l'hybridité constitutive de la diaspora africaine. C'est précisément ce lien entre Amérique et Afrique, Europe et Caraïbes, passé et présent, qui est opéré dans Sankofa: Mona/Shola est propulsée dans un espace hybride dans lequel s'unifie la diaspora africaine. Le public noir est donc invité à partager l'expérience de Mona et à accomplir le même voyage spirituel, qui consiste à connaitre le passé noir (celui de la traite et de l'esclavage) pour mieux appréhender l'avenir, tel un oiseau sankofa. Les gros plans sur le visage de Mona, associés à la voix off, offrent une incursion dans l'intimité du personnage. Le bateau qui emmène Mona/Shola de l'Afrique à l'Amérique reste invisible. Pourtant, c'est précisément le bateau négrier qui constitue pour Paul Gilroy le "chronotope des carrefours » de l'expérience noire de la souffrance, de l'arrachement et de la violence (Gilroy 199, cité par Letort 2). Du voyage négrier, il ne reste dans Sankofa que le vautour, symbole des oiseaux de proie qui suivaient les bateaux du commerce triangulaire à l'affût des cadavres jetés par-dessus bord. En réalité, l'expérience fondatrice de Shola se produit lorsque son corps est marqué au fer dans le fort. L'existence de la diaspora noire est construite dans ce marqueur spatio-temporel du fort, puis dans l'espace extradiégétique du voyage entre l'Afrique et l'Amérique.

\section{Renverser les images}

8 Haile Gerima ajoute une étape supplémentaire à la désorientation initiale du spectateur, qui correspond à l'arrachement de Mona à sa culture africaine, avec le 
renversement des images traditionnellement transmises par le cinéma, notamment hollywoodien. Gerima a ainsi déclaré dans une interview, à propos de Sankofa :

In Hollywood, most slaves are happy. They talk the same. Their identity is fully determined by the context of the plantation. They are nothing. They are property utilized to make the plantation life better, and they have no human dimensions, no desires. And this stereotype does not operate solely against Africans: the experiment began with Native Americans. So, the whole idea of Africans who are happy to be slaves, devoted to their owners, who will sell themselves twenty times over to save their gambling master-all this is the romantic literature of the plantation, the view from the plantation owner's perspective.

Now, what I did was flip this. I brought out the individual identities and motives of the characters, transforming the "happy slaves" into an African race opposed to this whole idea, by making the history of slavery full of resistance, full of rebellion. Resistance and rebellion-the plantation school of thought believed it was always provoked by outsiders, that Africans were not capable of having that human need. (Woolford 92)

De fait, aucun personnage d'esclave dans Sankofa n'est présenté comme "heureux d'être esclave ». L'essence même du personnage de Shango est de se rebeller et de résister, et en cela il correspond à cette description de Frantz Fanon dans Peau noire, masques blancs:

Pour le nègre qui travaille dans les plantations de canne du Robert [commune de la Martinique], il n'y a qu'une solution : la lutte. Et cette lutte, il l'entreprendra et la mènera non pas après une analyse marxiste ou idéaliste, mais parce que, tout simplement, il ne pourra concevoir son existence que sous les espèces d'un combat mené contre l'exploitation, la misère et la faim. (Fanon 181-182)

Tout au long du film, Shango est coiffé, comme tous les marrons du film, d'un foulard rouge qui symbolise sa défiance et chaque séquence le présente dans un acte de rébellion. Dans sa toute première séquence, il demande à Shola d'empoisonner toute la maisonnée, ce qui comprend les esclaves de maison, soupçonnés d'être parties prenantes de la domination blanche. Quant à Nunu, elle défie les maîtres par son refus de diluer son identité dans celle créée par les Blancs. Ce qui la constitue, c'est sa résistance à l'idéologie blanche. À l'origine, Nunu était un personnage essentiel du script de Gerima, écrit dès 1975 : elle en était l'héroïne, en tant qu'esclave dans une plantation de canne à sucre dans le Sud des États-Unis quelques années avant la guerre de Sécession (Pfaff 144).

Nunu et Shango amènent progressivement Mona à retrouver son identité africaine, en favorisant son retour aux rites des origines et en l'incitant à se rebeller contre le maître qui veut la violer. L'idée que la résistance est constitutive de l'existence est énoncée dans la séquence où Nunu réconforte un des esclaves contremaîtres; celui-ci lui répond: "Not being able to say no is what is killing me », ce qui sous-entend que la résistance permet de vivre. Quant à Joe, le mulâtre, qui est le personnage le plus susceptible d'être gagné à l'idéologie blanche, il sombre progressivement dans la folie et tue à la fois sa mère, Nunu, et son père, Father Raphael (Reginald Carter), le prêtre blanc. Ces derniers actes lui permettent de résister à l'endoctrinement du prêtre en faisant sienne la religion catholique, et surtout en se l'appropriant au prisme de son expérience de mulâtre : le film prend soin de multiplier les plans sur des images de madones blanches, qui n'ont pas de lien avec Joe, ce dont il se rend compte en tuant sa propre mère. Lorsque le prêtre blanc cherche à empêcher Joe de déposer le corps de Nunu sur l'autel de l'église, Joe s'écrie "Ain't she a saint? ", signifiant par là même sa résistance à une forme de religion qui exclut plutôt qu'elle n'inclut, alors que le prêtre 
a sans cesse cherché à nier Nunu en la présentant comme païenne. Joe est un personnage ambivalent, antipathique, dont la fonction est de signifier l'internalisation de l'oppression et de montrer les effets de la colonisation sur l'esprit. Joe souffre de sa double conscience et cherche, tout au long de la narration, à se débarrasser du pan de cette conscience que la société blanche juge inacceptable. Le meurtre de Nunu et de Father Raphael, et son suicide dans l'église, révèlent que Joe ne parvient pas à s'affranchir de l'oppression, contrairement à Mona, qui a accepté de se tourner vers les horreurs du passé pour reprendre possession de son avenir.

Le renversement des images inclut un renversement des tropes hollywoodiens. Ainsi la théoricienne radicale noire bell hooks indique que Shola correspond au stéréotype de la séductrice Jézabel et Nunu à celui de la réconfortante mammy, mais de fait ces stéréotypes sont renversés (hooks 127 ; Bogle). Shola est loin d'être une Jézabel qui tire parti de l'attrait qu'elle exerce sur les hommes blancs: le film montre au contraire qu'elle est à plusieurs reprises brutalement violée par son maître, ce qui permet au film de rompre le silence du cinéma hollywoodien à ce sujet. Les relations sexuelles forcées entre maîtres blancs et esclaves noires ont rarement été montrées à l'écran avant Sankofa - le film d'exploitation Mandingo, dont Quentin Tarantino s'est inspiré pour Django Unchained (2012), faisant figure d'exception. Nunu n'est pas non plus une mammy serviable et dévouée à sa famille blanche d'adoption: elle n'a au contraire aucun lien avec elle dans l'espace diégétique. Si Nunu est une mammy, c'est plutôt dans le sens de la Mère Afrique, alors que Shola la décrit ainsi : "She's all strength and passionate and tenderness ». Nunu n'est pas une mammy hollywoodienne, page blanche sur laquelle s'écrit l'histoire de la famille blanche, car elle porte en elle toute l'histoire de l'Afrique, qu'elle restitue, tel le griot, à la communauté africaine exilée sous forme de contes, de rituels et de paraboles ${ }^{4}$. Quant à Joe, figure de mulâtre tragique, néanmoins atypique car, contrairement à la plupart des films hollywoodiens, il s'agit dans le film de Gerima d'un homme et non d'une femme ${ }^{5}$, il renverse le mythe de l'oncle Tom. Le film montre délibérément Joe se réfugier dans la religion chrétienne, mais alors que l'oncle Tom est présenté comme une figure du martyr christique dans le roman d'Harriet Beecher Stowe, Joe démontre toute l'ironie d'un christianisme dont la norme est la blancheur et qui ne perçoit la sainteté qu'en blanc (Williams). À la place, Sankofa introduit une religion africaine, avec ses propres rites et sa propre culture : le film multiplie les images de cérémonies et de baptêmes. Ces nouveaux rites de la résistance éclairent d'une lueur nouvelle l'admonition de Sankofa au début du film : "Spirit of the dead, rise up! " La résistance permet à la spiritualité africaine de prendre le dessus sur l'oppression chrétienne, en réintroduisant le motif de la possession (« possess your bird of passage »), répété dans la possession de Mona par Shola. Il s'agit de désigner le christianisme comme l'un des grands oppresseurs des peuples africains et l'un des plus grands mensonges du colonialisme, selon le film : prétendant élever les Africains à la spiritualité, le colonialisme a pu occulter les pratiques religieuses préexistantes (Ukadike, 2014 184-185). Les personnages sont à la fois des individus, avec leurs motivations propres, et une communauté africaine, unie dans son élan de résistance.

\section{L'écriture symbolique}

11 Dans « Black American Cinema: The New Realism », Manthia Diawara décrit le nouveau cinéma des réalisateurs noirs américains indépendants dans les termes suivants : 
These films are concerned with the specificity of identity, the empowerment of Black people through mise-en-scène, and the rewriting of American history. Their narratives contain rhythmic and repetitious shots, going back and forth between the past and the present. Their themes involve Black folklore, religion, and the oral traditions which link Black Americans to the African diaspora. The narrative style is symbolic. (Diawara 10)

De fait, outre les liens effectués entre le passé et le présent, et une esthétique spécifique, Haile Gerima use de l'écriture symbolique, notamment avec le motif de l'oiseau sankofa. La première séquence du film indique que ce mot vient de l'akan, un dialecte ouest-africain, et qu'il signifie « one must return to the past in order to move forward ». Le sankofa est ce fameux «bird of passage » que les Africains et les Noirs américains doivent reconquérir dans le discours du prologue. Le film débute donc sur l'image du sankofa, cet oiseau dont la tête est tournée vers l'arrière, le buste vers l'avant, portant un œuf dans son bec, et se termine sur cette même image, comme pour signifier que le début est la fin et inversement, tout en indiquant que le début contient la fin, avec la symbolique de l'œuf. Le sankofa possède également une forme de cœur, ce qui ajoute la dimension supplémentaire des origines de l'existence.

Dans le dossier de presse rédigé spécialement pour la diffusion du film dans les salles de cinéma françaises en 2000, Haile Gerima écrit :

Dans Sankofa, le symbole récurrent de l'oiseau est une donnée primordiale. Au bout de vingt ans de recherche, j'ai fini par découvrir que l'oiseau était important aux yeux des esclaves et des "marrons ». Le vautour symbolise la dualité Vie/Mort. Pour les fugitifs, le vautour était un guide vers les collines, loin des chiens, des chevaux et des poursuivants. Quant à ceux qui succombaient, les vautours les dévoraient. Mais, dans ce cas, une symbolique supplémentaire opérait pour les Africains, celle de la liberté, car leur croyance voulait que le vautour ramenât l'esprit du défunt aux sources de la Mère Afrique. Certains mythes mentionnaient même des esclaves échappés qui, mutés en oiseaux, s'étaient envolés pour l'Afrique. (Gerima, 2000)

Or il s'agit exactement du parcours de Mona, qui se transforme, à la suite de son acte ultime de rébellion - la mise à mort du maître -, en oiseau s'envolant pour l'Afrique.

En réalité, dès le prologue, l'accent est mis sur l'écriture symbolique, à mesure que la voix off nomme ceux qui doivent se sentir concernés par le voyage du sankofa :

Spirits of the dead, rise up, lingering spirits of the dead, rise up and possess your bird of passage. Those stolen Africans step out of the ocean from the wombs of the ships and claim your story. [...] Those Africans, shackled in leg irons and enslaved, step out of the acres of cane fields and cotton fields and tell your story. [...] Those lynched in the magnolias, swinging on the limbs of the weeping willows, rotting food for the vultures, step down and claim your story. [...] Those tied, bound, and whipped from Brazil to Mississippi, step out and tell your story. Those in Jamaica, in the fields of Cuba, in the swamps of Florida, the rice fields of Carolina-you waiting Africans, step out and tell your story.

L'incantation du sorcier Sankofa dans le fort d'Elmina rassemble toutes les origines partagées de la diaspora africaine, origines qui ont été oubliées par les êtres comme Mona au sortir du mouvement pour les droits civiques, mais aussi par les autorités africaines, incarnées dans le film par les soldats du fort, qui chassent le sorcier. Dans cette écriture symbolique, chaque être humain noir vient de toutes ces contrées où les Noirs ont été forcés de ramasser le coton ou de couper la canne à sucre, ont été lynchés, pendus et fouettés, de l'Amérique Latine au Sud des États-Unis.

Mona symbolise tous les Noirs qui ont oublié ces origines, ce qui les aliène : sa séquence de présentation montre ainsi qu'elle est aliénée par le regard du photographe, 
incarnation des médias dominants aux États-Unis. Mona est piégée dans une logique d'oppression, marquée dans l'esthétique du film par une succession de plans en plongée sur le corps de Mona et en contre-plongée sur le photographe. L'oiseau sankofa lui permet alors d'exister parce qu'il la transforme en être capable de résister, par exemple lorsque Shango place un pendentif de sankofa autour de son cou: «After he put that bird around my neck, I became a rebel. No longer was I scared to be flogged and burned.» Évidemment, la séquence de prédation du photographe blanc est aussi un commentaire métatextuel sur la prédation institutionnelle opérée par la caméra hollywoodienne sur les actrices noires, auxquelles on a souvent demandé - comme on l'a suggéré plus haut - d'être des Jézabel (ce qui fut le cas de l'actrice noire américaine Fredi Washington) ou au contraire des êtres asexués dévoués à leurs maîtres blancs, telle l'actrice Hattie McDaniel, la mammy d'Autant en emporte le vent, qui dans le même temps qu'elle recevait l'Oscar du meilleur second rôle féminin devait s'asseoir à une table séparée des acteurs blancs du film lors de la cérémonie. Cette prédation est soulignée par les plans en plongée et par le fait que la caméra se substitue à l'œil du photographe.

\section{Le mouvement du sankofa}

15 La quatrième étape de la création d'une esthétique de la résistance dans le film de Gerima procède directement de l'écriture symbolique, liée au mouvement du sankofa. De fait, le film sollicite à de nombreuses reprises le motif de la boucle ouverte: les séquences de début semblent être les séquences de fin et de nombreuses séquences s'organisent selon une disposition concentrique, comme le signale Anne Crémieux dans son livre sur les cinéastes noirs américains (Crémieux, 2004 181). Ainsi lors de la préparation de la rébellion, ou lorsque Nunu raconte des histoires aux enfants, ou encore lorsque les esclaves entourent une esclave enceinte sur le point d'accoucher, les esclaves forment des cercles qui excluent les Blancs et protègent leur centre. Le cercle permet tantôt de protéger l'existence même des esclaves (dans le cas du bébé à naître), tantôt de protéger leur culture africaine (dans le cas des histoires de Nunu). Haile Gerima a appelé ce type de disposition le «cinéma du foyer »(fireplace cinema). Il explique la centralité du foyer dans la culture africaine, foyer qui permet de rassembler, de réchauffer, de partager :

The fireplace tradition created time and space for observation and critical analysis, benefiting the entire social network, including artists. It is entirely appropriate to perceive the cinema as being symbolic of the fire-the central point of life, earth and energy. (Gerima, 1984 54)

16 Ces moments de disposition concentrique, autour d'un foyer ou d'un enfant à naître, fonctionnent donc non seulement comme des actes de résistance au niveau de la narration, mais aussi comme des commentaires métatextuels sur la fonction de résistance du cinéma : le spectateur fait partie intégrante du cercle autour du foyer. Ces séquences invitent le spectateur à observer et à analyser, de la même manière que Shola observe et analyse : nous devons effectuer le même voyage spirituel que Mona/ Shola, un retour vers le passé pour mieux appréhender l'avenir. De fait, le mouvement du sankofa - et donc du film - n'est pas exactement circulaire, bien qu'il puisse de prime abord paraître tel. Lorsque Mona revient, elle est irrémédiablement changée. Elle ne répond plus aux appels du photographe, car elle n'est plus un objet ; elle est devenue l'agent de son histoire, et peut désormais regarder l'avenir, avec sa communauté, tout 
en intégrant le passé dans sa conscience. Il en va de même du cinéma noir américain selon Haile Gerima : tel le sankofa, il avance tout en ayant pris conscience de ses formes antérieures.

\section{BIBLIOGRAPHIE}

BOGLE, Donald. Toms, Coons, Mulattoes, Mammies, and Bucks: An Interpretive History of Blacks in American Films. New York : Viking Press, 1973.

CRÉMIEUX, Anne. Les Cinéastes noirs américains et le rêve hollywoodien. Paris : L'Harmattan, 2004.

CRÉMIEUX, Anne. « Haile Gerima, une œuvre entre deux continents ». Africultures, avril 2009. Page consultée le 15 mars 2018.

DIAWARA, Manthia. «Black American Cinema: The New Realism ». Black American Cinema. Dir. Manthia Diawara. New York : Routledge, 1993, p. 3-25.

DU BOIS, W.E.B. The Souls of Black Folk: Essays and Sketches. Chicago : A.C. McClurg \& Co., 1903.

FANON, Frantz. Peau noire, masques blancs. Paris : Éditions du Seuil, 1952.

FIELD, Allyson Nadia, Jan-Christopher HORAK et Jacqueline Najuma STEWART, dir. LA Rebellion: Creating a New Black Cinema. Oakland : University of California Press, 2015.

GERIMA, Haile. Dossier de presse de Sankofa rédigé à l'occasion de la sortie du film en France, archives personnelles de l'autrice, 2000.

GERIMA, Haile. « Fireplace-Cinema (Gathering, Warming, Sharing) ». Filmfaust, n 39, 1984, p. 44-55.

GUEDJ, Pauline. Panafricanisme, religion akan et dynamiques identitaires aux États-Unis. Le chemin du Sankofa. Paris : L’Harmattan, 2011.

GUERRERO, Ed. Framing Blackness: The African American Image in Film. Philadelphie : Temple University Press, 1993.

hooks, bell. Reel to Real: Race, Sex, and Class at the Movies. New York : Routledge, 2009.

KANDÉ, Sylvie. « Look Homeward, Angel: Maroons and Mulattos in Haile Gerima’s Sankofa ». African Cinema: Postcolonial and Feminist Readings. Dir. Kenneth W. Harrow. Trenton : Africa World Press, 1999, p. 89-114.

LETORT, Delphine. «Sankofa (Haile Gerima, 1993) : la mémoire de l'esclavage dans la conscience diasporique ». Transatlantica, $\mathrm{n}^{\circ}$ 2, 2012. Page consultée le 15 mars 2018.

MURPHY, Joseph M. Santería: African Spirits in America. Boston : Beacon Press, 1993.

PFAFF, Françoise. Twenty-five Black African Filmmakers: A Critical Study, with Filmography and Biobibliography. New York: Greenwood Press, 1988.

REID, Mark A. Black Lenses, Black Voices: African American Film Now. Lanham : Rowman \& Littlefield, 2005.

«Story of LA Rebellion (the) ». UCLA Film and Television Archive. Page consultée le 15 mars 2018. 
TAYLOR, Clyde. «The LA Rebellion: New Spirit in American Film ». Black Film Review, vol. 2, $\mathrm{n}^{\circ}$ 2, 1986, p. 9-16.

UKADIKE, Nwachukwu Frank. « Critical Dialogues: Transcultural Modernities and Modes of Narrating Africa in Documentary Films ». Critical Approaches to African Cinema Discourse. Dir. Nwachukwu Frank Ukadike. Lanham : Lexington Books, 2014, p. 177-191.

UKADIKE, Nwachukwu Frank. Questioning African Cinema: Conversations with Filmmakers. Minneapolis : University of Minnesota Press, 2002.

WALL, David C., et Michael T. MARTIN, dir. The Politics and Poetics of Black Film: Nothing But a Man. Bloomington : Indiana University Press, 2015.

WEINER, Rex. « Straight as an Arrow ». Daily Variety, 25 juillet 1995, 17.

WILLIAMS, Linda. Playing the Race Card: Melodramas of Black and White from Uncle Tom to O.J. Simpson. Princeton : Princeton University Press, 2001.

WOOLFORD, Pamela. « Filming Slavery: A Conversation with Haile Gerima ». Transition, $\mathrm{n}^{\circ} 64$, 1994, p. 90-104.

\section{NOTES}

1. Les « retours » à Oscar Micheaux sont multiples dans la carrière d'Haile Gerima : l'un de ses premiers films, Harvest: 3,000 Years (1976), reçut le prix Oscar Micheaux du Black Filmmakers Hall of Fame, à Oakland, en Californie. Dans un entretien avec Françoise Pfaff en 1977, il tenait les propos suivants : "In order to survive, young black filmmakers should follow the example of early Black American directors like Oscar Micheaux. Even if one is critical about the content of his films, one should learn from him » (Pfaff 142).

2. Pauline Guedj indique dans son ouvrage sur la mythologie akan que l'oiseau sankofa - « un symbole akan, très populaire au Ghana, représentant un oiseau se penchant sur sa queue, métaphore de la nécessité pour tout homme de se tourner vers son passé pour avancer vers le futur » (Guedj 9) - est devenu aux États-Unis dans les années 1960 et 1970 l'emblème de toute une génération de militants qui préconisaient le retour aux racines africaines pour décoloniser les esprits et guérir la communauté noire américaine des méfaits de l'oppression.

3. « La voix off s'adresse à la diaspora noire des Amériques dans un discours englobant qui efface les limites géographiques entre pays esclavagistes pour décrire une communauté de victimes parmi les Noirs de la traite atlantique » (Letort 7).

4. Le personnage de Nunu est très probablement inspiré de la mère et de la grand-mère éthiopiennes de Gerima : « His mother and grandmother were great storytellers, and listening to them, he acquired mastery of the legends and tales of the Ethiopian oral tradition, which was later to become the quintessence of his cinematic narrative style » (Ukadike, 2002 253).

5. La mulâtresse tragique est une figure importante du cinéma hollywoodien lorsque celui-ci se préoccupe de questions raciales, par exemple dans Images de la vie (Imitation of life, John Stahl, 1934, dont Douglas Sirk a réalisé en 1959 un remake intitulé en français Mirage de la vie), L'Héritage de la chair (Pinky, Elia Kazan, 1949) ou encore L'Esclave libre (Band of Angels, Raoul Walsh, 1957). Il existe peu de mulâtres masculins dans le cinéma hollywoodien. Silas Lynch, le mulâtre de Naissance d'une nation, n'est pas tragique mais maléfique. 


\section{RÉSUMÉS}

Le cinéaste éthiopien indépendant Haile Gerima fait partie du groupe de réalisateurs noirs américains baptisé LA Rebellion. Parallèlement à sa carrière d'universitaire, Gerima a réalisé plusieurs films traitant de divers aspects de l'expérience noire américaine et diasporique. C'est avec Sankofa (1993) qu'il connut son premier grand succès. Traitant de la résistance à l'oppression par le biais de la mythologie akan - en particulier l'oiseau sankofa, symbole de la nécessité pour toute personne de se tourner vers son passé pour appréhender l'avenir -, le film définit une politique et une esthétique de la résistance à l'esclavage. Sankofa a essentiellement été analysé dans sa dimension politique, idéologique et historique. Cet article se penche sur sa dimension esthétique et sémiotique, élément à part entière du discours idéologique sur la résistance à l'esclavage.

Haile Gerima, an independent Ethiopian filmmaker who belonged to the LA Rebellion film movement, directed films which dealt with various aspects of the African American and black diasporic experience. Sankofa (1993) was his first important critical and commercial success. The film used Akan mythology-in particular the sankofa bird symbolizing the need for every person to turn to the past in order to face their future-to broach the issue of resistance to oppression. In Sankofa, Gerima developed a politics and aesthetic of resistance. While the film's political, ideological, and historical dimensions have been extensively analyzed, this essay focuses on its aesthetic dimension, which is the cornerstone of its ideological discourse on resistance to slavery.

\section{INDEX}

Keywords : Sankofa, Haile Gerima, slavery, resistance, LA Rebellion

Mots-clés : Sankofa, Haile Gerima, esclavage, résistance, LA Rebellion

\section{AUTEUR}

\section{CLAIRE DUTRIAUX}

Université Paris Sorbonne 